\title{
Near Constant Envelope Trellis Shaping for PSK Signaling
}

\author{
Makoto Tanahashi* and Hideki Ochiai ${ }^{\dagger}$ \\ Department of Electrical and Computer Engineering, \\ Yokohama National University \\ 79-5 Tokiwadai, Hodogaya, Yokohama, Kanagawa 240-8501, Japan \\ Email: *makoto@ochiailab.dnj.ynu.ac.jp, †hideki@ynu.ac.jp \\ ${ }^{\dagger}$ Corresponding author
}

This paper was in part presented at the IEEE International Conference on Communications 2007 (ICC 2007), Glasgow, Scotland, June 2007.

\begin{abstract}
In this paper, we propose a powerful trellis shaping for peak-to-average power ratio (PAR) reduction of pulse-shaped phase shift keying (PSK) systems. The proposed approach consists of 1) memory extension of trellis shaping encoder for capturing the full effect of pulse shaping filter impulse response, and 2) simple branch metrics that quantify envelope fluctuation of the filter output. Simulation results demonstrate that achieving near constant envelope is possible even if a pulse shaping filter with roll-off factor as low as 0.1 is employed. Furthermore, due to the circular nature of the resulting signal trajectory, the proposed system also exhibits a robustness against sampling timing jitter at the receiver.
\end{abstract}

\section{Index Terms}

Band-limited single-carrier communications, peak-to-average power ratio reduction, trellis shaping

\section{INTRODUCTION}

Achieving high power amplifier efficiency has been one of the major issues in the communication system design. In many existing wireless communications standards, the application 
of costly linear power amplifier (PA) is difficult. Thus, constant envelope modulations such as offset quadrature phase shift keying (OQPSK) and continuous phase modulation (CPM) have been preferable choices as they can be operated with nonlinear PAs that have high direct-current (DC) to radio-frequency (RF) conversion efficiency. The price for the use of constant envelope modulations is its low bandwidth efficiency. They have difficulties in achieving a transmission rate of multiple bits per $\mathrm{Hz}$, which is readily achievable by linear modulations such as $M$-ary phase shift keying (PSK) and quadrature amplitude modulation (QAM).

On the other hand, linear modulations generate a signal with high peak-to-average power ratio (PAR). In particular, if one attempts to increase spectral efficiency by using high-order signal constellations or narrower pulse shaping filter, the resulting signal envelope tends to exhibit large dynamic range or high PAR. In order to amplify such a signal with high PAR, the use of linear PAs operated with a large back-off is necessary, which in turn results in prohibitively low amplifier efficiency.

The amplifier efficiency problem of single-carrier $M$-PSK systems may not be as severe as that of the orthogonal frequency division multiplexing (OFDM) or other multicarrier modulation systems where much attention has been paid in the recent literature. Nevertheless, achieving low PAR with strictly band-limited single-carrier signals has been an issue for decades. The PAR of such signals can be controlled by constraining transition patterns of successive symbols. The well-known classical approaches in this direction include $\pi / 4$-shift QPSK, which can be implemented with almost negligible increment of complexity. Since the constraint posed on the signal transition in this case is only over two adjacent symbols, its PAR controlling capability is limited. As a result, it is effective only at moderate values of roll-off factor $\alpha$. Furthermore, it is effective for QPSK scenario (i.e., two bits per symbol), but less effective for 8-PSK or higher-order PSK.

In [1], an adaptive PAR suppression algorithm has been proposed for pulse-shaped PSK modulations. The PAR reduction can be achieved by intentionally scaling the amplitude of the PSK symbols. This technique may be effective, but at the cost of the transmitter complexity and amplitude fluctuation sensitivity at the receiver. More recently, a new PAR reduction scheme based on the trellis structure of the pulse-shaping filter is proposed in [2]. By viewing the pulseshaping filter as a convolutional encoder and pruning the edges of the trellis that result in a high peak power, considerable PAR reduction is achieved. However, the complexity of constructing 
an encoder and decoding this convolutional code seems to remain an issue for this technique.

Another interesting trellis based approach is the use of trellis shaping (TS), originally proposed by Forney [3] for reduction of average power of high-order QAM signals. This technique has been successively applied to a PAR reduction of single carrier systems by Morrison [4] and further studied in [5]. More recently, it has been applied to the PAR reduction of multicarrier and OFDM systems in $[6,7]$. TS makes use of a convolutional coding structure for constraining transition of consecutive signal constellations. The results of [4], however, do not show significant improvement over $\pi / 4$-shift QPSK in terms of PAR reduction capability. The reason for this may stem from its empirical metric design used in the shaping process. Since the phase difference between two consecutive symbols is used for a criterion of code selection process, the PAR reduction capability of [4] is analogous to that of $\pi / 4$-shift QPSK system.

In this paper, we propose a new PAR reduction scheme for single-carrier PSK systems based on TS and demonstrate that a near constant envelope is achievable even if the pulse shaping filter with a roll-off factor as low as 0.1 is used. As a side effect, the generated signal turns out to exhibit robustness against the inter-symbol interference (ISI) associated with the sampling timing jitter at the receiver.

One major drawback of the proposed TS is its computational overhead. Similar to [1], the proposed TS actually calculates the band-limited baseband signal waveforms upon PAR reduction process. Another drawback is the requirement of additional constellation redundancy imposed by the shaping encoder. The conventional TS is employed typically with one-bit redundancy per symbol, which can achieve best performance in terms of average power reduction capability as reported in [8]. On the other hand, the reduction of dynamic range does not augment minimum Euclidean distance of signal constellations, and therefore, the SNR required for a certain error rate increases when the systems are compared under the condition of the same information rate. This loss in terms of SNR may limit applications of the TS, even though it can achieve significant amount of PAR reduction and thus increase amplifier efficiency. To mitigate these drawbacks, we also consider the use of multidimensional TS as in $[5,7]$. Compared to conventional TS with one-bit redundancy, with a slight decrease in the PAR reduction capability, the multidimensional TS can reduce not only the required constellation redundancy but also the required memories of shaping encoder. The latter fact substantially contributes to the reduction of the overall TS complexity. 


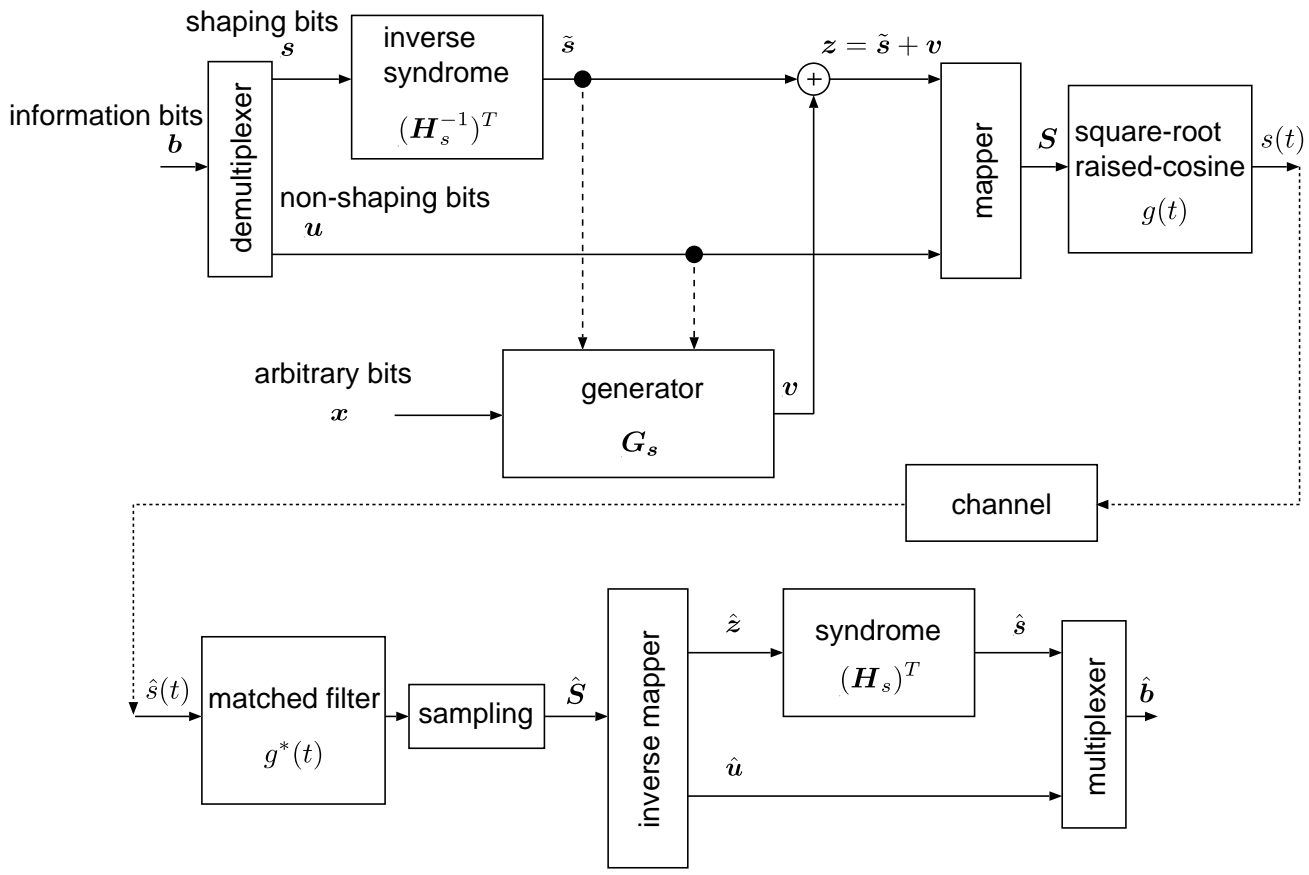

Fig. 1. General construction of TS and system model.

This paper is organized as follows. Section II is devoted to the system description as well as a brief review of notations used for the TS in the paper. Since the trellis shaping for PAR reduction in general introduces correlation among transmitted symbols, the average power of the signal after pulse shaping can vary depending on the generated symbol patterns even with PSK constellations. If so, this complicates a systematic derivation of shaping metrics. To deal with this problem, at the end of Section II, we also introduce a theorem which guarantees that the average power is invariant after pulse shaping as long as the filter is a commonly used square-root raised-cosine filter. In Section III, we propose a new TS design for PAR reduction. Section IV compares the proposed TS and conventional techniques through computer simulations. Finally, concluding remarks are given in Section V. For the purpose of comparison with $\pi / 4$-shift QPSK, we mainly focus on a shaping system with 8-PSK signaling. However, we note that the proposed scheme can be applied to a higher order PSK as well in a straightforward manner.

\section{SYSTEM DESCRIPTION}

The overall system considered in this paper is described in Fig. 1. The details of trellis shaping (TS) can be found in $[3,7,8]$. We here briefly describe the TS procedure and notations used in 
the paper.

\section{A. Trellis Shaped PSK System}

Trellis shaping makes use of the convolutional coding structure to generate constrained sequences. Let $\boldsymbol{G}_{s}$ denote a $1 \times n_{s}$ generator matrix of a binary convolutional code and $\boldsymbol{H}_{s}^{T}$ a corresponding $n_{s} \times\left(n_{s}-1\right)$ syndrome matrix which satisfies

$$
\boldsymbol{G}_{s} \boldsymbol{H}_{s}^{T}=\mathbf{0}
$$

where 0 is a zero row vector of length $n_{s}-1$. The left inverse of this syndrome matrix (hereafter simply called inverse syndrome) is denoted by $\left(\boldsymbol{H}_{s}^{-1}\right)^{T}$ and satisfies

$$
\left(\boldsymbol{H}_{s}^{-1}\right)^{T} \boldsymbol{H}_{s}^{T}=\boldsymbol{I}
$$

where $\boldsymbol{I}$ is an identity matrix.

The information bits $\boldsymbol{b}$ are divided into shaping bits $s$ and non-shaping bits $\boldsymbol{u}$. Given the above matrices, the former is encoded by the inverse syndrome to generate

$$
\tilde{\boldsymbol{s}}=\boldsymbol{s}\left(\boldsymbol{H}_{s}^{-1}\right)^{T} .
$$

Let us assume that virtual input bits $\boldsymbol{x}$ are fed to $\boldsymbol{G}_{s}$. The output codeword $\boldsymbol{v}=\boldsymbol{x} \boldsymbol{G}_{s}$ is then added (in a modulo-2 manner) to $\tilde{\boldsymbol{s}}$. The corresponding sequence

$$
\boldsymbol{z}=\tilde{\boldsymbol{s}}+\boldsymbol{v}
$$

and non-shaping bits $\boldsymbol{u}$ determine the signal constellation sequence $\boldsymbol{S}=\left\{\ldots, S_{-2}, S_{-1}, S_{0}, S_{1}, S_{2}, \ldots\right\}$.

The information sequence $s$ can be retrieved at the receiver by simply feeding $z$ into the syndrome as

$$
\boldsymbol{z} \boldsymbol{H}_{s}^{T}=(\tilde{\boldsymbol{s}}+\boldsymbol{v}) \boldsymbol{H}_{s}^{T}=\boldsymbol{s} \underbrace{\left(\boldsymbol{H}_{s}^{-1}\right)^{T} \boldsymbol{H}_{s}^{T}}_{\boldsymbol{I}}+\boldsymbol{x} \underbrace{\boldsymbol{G}_{s} \boldsymbol{H}_{s}^{T}}_{\mathbf{0}}=\boldsymbol{s}+\mathbf{0}=\boldsymbol{s} .
$$

Finally, the baseband signal at the transmitter is expressed in a non-causal form as

$$
s(t)=\sum_{l=-\infty}^{\infty} S_{l} g\left(t-l T_{s}\right),
$$

where $T_{s}$ is the Nyquist interval and $g(t)$ is the impulse response of a pulse shaping filter. Our objective here is to find a suitable arbitrary sequence $\boldsymbol{x}$ (or equivalently its corresponding 
codeword $\boldsymbol{v}$ ) with reference to given $\tilde{\boldsymbol{s}}$ and $\boldsymbol{u}$, such that the resulting complex baseband signal $s(t)$ is of low dynamic range. Since $\boldsymbol{v}$ is a codeword of a given convolutional code $\boldsymbol{G}_{s}$, the Viterbi algorithm (VA) can be used for codeword selection. The challenge is to develop a suitable metric associated with the trellis branch that can efficiently reduce the signal PAR.

\section{B. Multidimensional Trellis Shaping}

The codeword sequence $\boldsymbol{v}$ chosen by the VA based on $\boldsymbol{G}_{s}$ has $n_{s}$ bits per trellis section. Suppose that each $n_{s}$-tuple of the binary codeword $\boldsymbol{v}$ simultaneously controls $d$ successive symbol outputs, each consisting of signal constellation with $M$ points. In other words, since inverse syndrome $\left(\boldsymbol{H}_{s}^{-1}\right)^{T}$ is an $\left(n_{s}-1\right) \times n_{s}$ matrix, we impose one bit redundancy for every $d$ symbols. In this case, $d$ is called shaping dimensionality, representing the length of symbols proceeded at each trellis section. Similar to the concept of multidimensional trellis coded modulation (TCM), the TS with $d>1$ is generally referred to as multidimensional shaping [5,7].

It can readily be seen that for a given symbol mapping with $M$ constellation points, the value $n_{s}$ can be chosen arbitrarily within the range of

$$
1<n_{s} \leq d \log _{2} M
$$

The resulting information rate after shaping, denoted by $R_{s}$ in [bit/symbol], is given by

$$
R_{s}=\frac{1}{d}(\underbrace{d \log _{2} M-n_{s}}_{\text {non-shaping bits }}+\underbrace{n_{s}-1}_{\text {shaping bits }})=\log _{2} M-\frac{1}{d} .
$$

Hence, if 8-PSK signal is shaped with $d=1$ and $d=2$, then the information rates $R_{s}$ are 2 and 2.5 [bit/symbol], respectively. It should be noted that $R_{s}$ does not depend on the choice of $n_{s}$.

In the subsequent sections, for notational convenience we group the $d$-tuple of transmitted symbols and write

$$
\boldsymbol{S} \triangleq\left\{\ldots, \boldsymbol{S}_{-1}, \boldsymbol{S}_{0}, \boldsymbol{S}_{1}, \ldots\right\}
$$

where $\boldsymbol{S}_{l}=\left\{S_{d l}, S_{d l+1}, \cdots, S_{d l+d-1}\right\}$. Since we restrict our attention to the case of $M$-ary PSK, $S_{l}$ takes a value from a set of $M$-ary PSK constellations denoted by $\mathcal{S}=\left\{e^{j 2 \pi m / M} \mid m=\right.$ $0,1, \ldots, M-1\}$. 


\section{Partial Signal Representation}

Referring to (6), we assume that $g(t)$ sustains non-zero amplitude over $K_{s}$ symbol intervals and has a negligible effect elsewhere, i.e.,

$$
g(t) \approx 0, \quad \text { for } \quad t<-\frac{K_{s}}{2} T_{s}, \quad \frac{K_{s}}{2} T_{s} \leq t
$$

Without loss of generality, we also assume that $K_{s}$ is restricted to be an even number. For discretetime notation, we sample $s(t)$ with a time interval $\Delta t=T_{s} / N_{s}$, where $N_{s}$ is an oversampling factor. The desired discrete sample representation of the signal (6) $s[n] \triangleq s(n \Delta t)$ is then given by

$$
s[n]=\sum_{k=-\infty}^{\infty} A_{k} g[n-k]
$$

where $g[n] \triangleq g(n \Delta t)$ and

$$
A_{k} \triangleq \begin{cases}S_{k / N_{s},} & k \text { is a multiple of } N_{s}, \\ 0, & \text { otherwise. }\end{cases}
$$

Let $s_{l}[\tilde{n}]$ denote the sample signal $s[n]$ truncated for one Nyquist symbol interval from $n=$ $\left(l-\frac{K_{s}}{2}\right) N_{s}$, i.e.,

$$
s_{l}[\tilde{n}] \triangleq s\left[\tilde{n}+\left(l-\frac{K_{s}}{2}\right) N_{s}\right] \quad \text { for } 0 \leq \tilde{n}<N_{s},
$$

where $l$ corresponds to an index of the current symbol. The truncated part $s_{l}[\tilde{n}]$ will be referred to as a partial signal in what follows. Substituting (11) and (12) into (13) and after some manipulation, we obtain

$$
s_{l}[\tilde{n}]=\sum_{k=-K_{s}+1}^{0} S_{l+k} g\left[\tilde{n}-\left(k+\frac{K_{s}}{2}\right) N_{s}\right], \quad \tilde{n}=0,1, \ldots, N_{s}-1 .
$$

Apparently, $s_{l}[\tilde{n}]$ depends on the $l$ th symbol $S_{l}$ and its $\left(K_{s}-1\right)$ previous symbols. Therefore, for given $\left(K_{s}-1\right)$ previously determined symbols $\left\{S_{l-\left(K_{s}-1\right)}, \ldots, S_{l-1}\right\}$ and current input data bits denoted by $\boldsymbol{b}_{l}$, our goal is to successively map $\boldsymbol{b}_{l}$ to $S_{l}$ using the TS structure such that the corresponding partial signal has a low PAR.

The associated instantaneous power normalized by its average power can be defined as

$$
p_{l}[\tilde{n}]=\frac{1}{P_{a v}}\left|s_{l}[\tilde{n}]\right|^{2},
$$


where $P_{a v}$ is the average power after shaping. Since the TS can be seen as an instance of modulation codes, it introduces correlation into the transmitted symbol sequence $S$. Therefore, even if each symbol $S_{l}$ has a unit energy, the corresponding sequence does not necessarily guarantee a unit average power after pulse shaping. In other words, the average power after pulse shaping $P_{a v}$ does not necessarily match with $E\left[\left|S_{l}\right|^{2}\right] / T_{s}$ and is not known until the shaped sequence $s[n]$ is generated. As will be shown in the next section, however, the proposed TS makes use of (15) for metric calculation of each trellis section. Thus, if $P_{a v}$ is subject to fluctuation depending on the shaped symbols, the shaping process also needs to estimate and adjust the resulting average power upon calculating (15). This dependence renders systematic metric design difficult, considering that the PAR itself is a function of the average power.

Fortunately, the following theorem holds for some pulse shaping filters.

Theorem 1: Let $\left\{S_{l}\right\}$ denote transmitting symbols and $g(t)$ denote the impulse response of the pulse shaping filter. The average power of the signal after the pulse shaping filter $s(t)$ defined by (6) is not affected by the shape of the autocorrelation function of $\left\{S_{l}\right\}$ if both the following conditions are satisfied:

1) $g(t)$ is an even function and has a finite energy.

2) the function $g(t) * g(t)$, where $*$ denotes convolution, satisfies Nyquist condition for the zero ISI [9].

Proof: See the Appendix.

Note that commonly used matched filter systems, operating with a square-root raised-cosine function for $g(t)$, satisfy the above conditions. Therefore, one can always assume that the average power is constant upon designing the trellis shaping metrics.

\section{Proposed TREllis Shaping FOR PAR REDUCTION}

The proposed TS consists of memory extension and simple branch metrics. By extending the memory of shaping encoder, the full effect of pulse shaping filter impulse response can be captured upon codeword selection. On the other hand, suitable branch metrics are required for quantifying envelope fluctuation of the instantaneous power of the partial signal $p_{l}[\tilde{n}]$. These issues are detailed in what follows. 


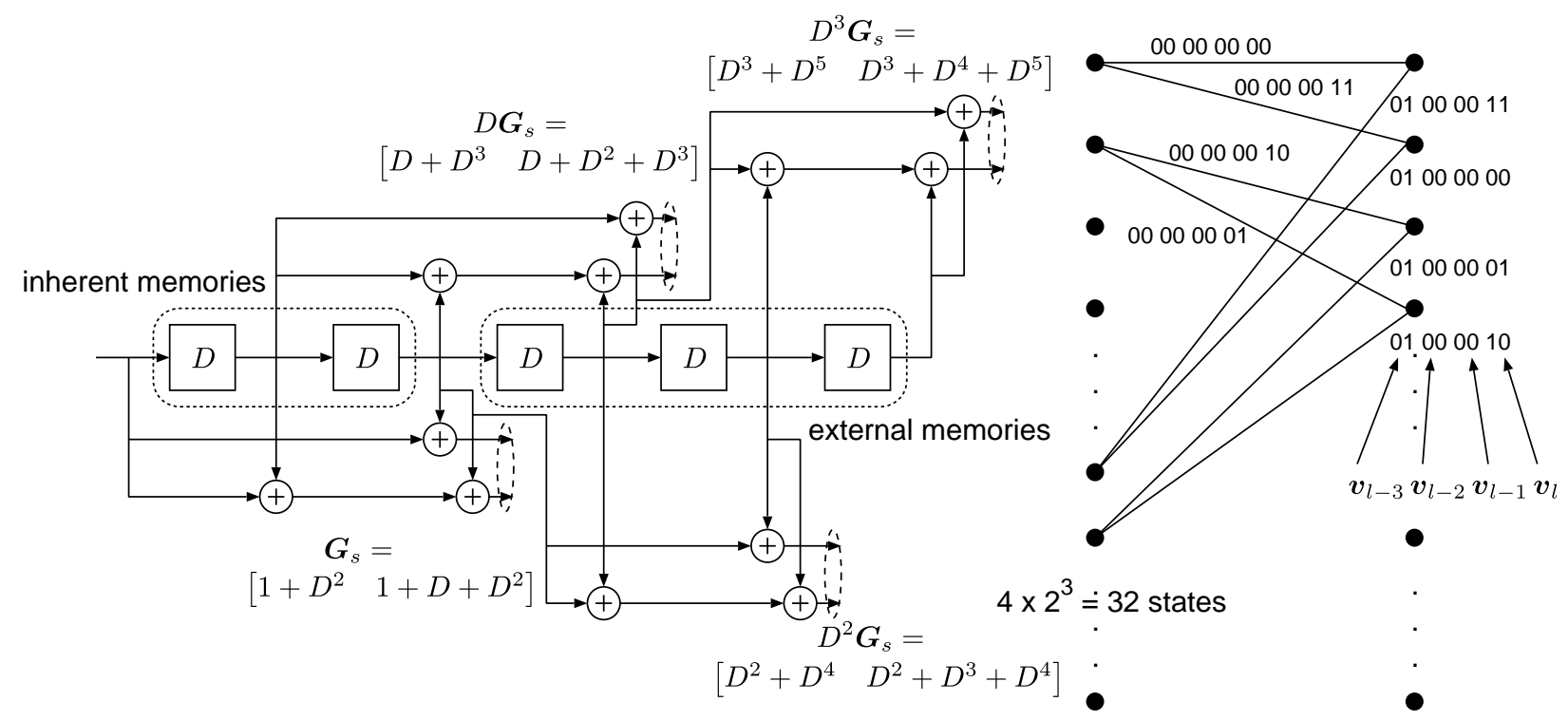

Fig. 2. An example extended memory encoder with $\nu=2$ and $m_{e x}=3$ and its trellis diagram.

\section{A. Memory Extension}

In the TS framework, we wish to assign each trellis section with $d$ (single or multiple) transmitting symbols as discussed in Section II-B. Let $\tilde{\boldsymbol{s}}_{l}$ and $\boldsymbol{u}_{l}$ denote the shaping and nonshaping bits corresponding to the $l$ th trellis section, respectively, that form the $l$ th group of transmitted symbols $\boldsymbol{S}_{l}$. Since $\tilde{\boldsymbol{s}}_{l}$ and $\boldsymbol{u}_{l}$ are uniquely determined by the associated $l$ th information bits $\boldsymbol{b}_{l}$, the only part that the TS process can control for the partial signal generation is the codeword bits $\boldsymbol{v}_{l}$ and its $\left\lceil K_{s} / d\right\rceil-1$ previous counterparts, where $\lceil\xi\rceil$ denotes the greatest integer not greater than $\xi$. Hence, for given information bits, the $l$ th symbol block $\boldsymbol{S}_{l}$ should be determined by $\boldsymbol{v}_{l}, \ldots, \boldsymbol{v}_{l-\left(\left\lceil K_{s} / d\right\rceil-1\right)}$. Therefore, it is desirable that the $l$ th trellis section should include all its associated previous outputs required to calculate $p_{d l}[\tilde{n}], p_{d l+1}[\tilde{n}], \cdots, p_{d l+d-1}[\tilde{n}]$. This can be done by expanding the states of the trellis diagram.

To this end, Morrison [4] proposed to attach an external memory to the shaping encoder $\boldsymbol{G}_{s}$. Specifically, suppose that a binary convolutional encoder $\boldsymbol{G}_{s}$ has a memory of $\nu$ bits and consider a bit sequence $\boldsymbol{x}=\left\{\ldots, x_{l-\nu}, x_{l-\nu+1}, \ldots, x_{l}, \ldots\right\}$ that forms a codeword sequence $\boldsymbol{v}=\left\{\ldots, \boldsymbol{v}_{l}, \ldots\right\}$. Here, the $l$ th codeword bits $\boldsymbol{v}_{l}$ and $x_{l}$ are related by

$$
\boldsymbol{v}_{l}=x_{l} \boldsymbol{G}_{s} .
$$


Note that since $\boldsymbol{G}_{s}$ has $\nu$-bit memory, $\boldsymbol{v}_{l}$ is a function of $\nu+1$ successive inputs, or equivalently, the states defined by $\Gamma=\left[\begin{array}{llll}x_{l} & x_{l-1} & \cdots & x_{l-\nu}\end{array}\right]$. Then the previous output symbol of this codeword is expressed by using a delay operator $D$ as

$$
\boldsymbol{v}_{l-1}=x_{l-1} \boldsymbol{G}_{s}=D x_{l} \boldsymbol{G}_{s}=x_{l} \cdot D \boldsymbol{G}_{s}
$$

Therefore, the convolutional encoder with generator matrix consisting of $\boldsymbol{G}_{s}$ and $D \boldsymbol{G}_{s}$ can simultaneously generate $\boldsymbol{v}_{l}$ and $\boldsymbol{v}_{l-1}$ as its outputs. In other words, the $l$ th output of an extended generator matrix $\left[\begin{array}{ll}\boldsymbol{G}_{s} & D \boldsymbol{G}_{s}\end{array}\right]$ is given by $\left[\begin{array}{ll}\boldsymbol{v}_{l} & \boldsymbol{v}_{l-1}\end{array}\right]$, and the corresponding trellis state for this output pair is given by $\Gamma=\left[\begin{array}{lllll}x_{l} & x_{l-1} & x_{l-2} & \cdots & x_{l-(\nu+1)}\end{array}\right]$. Likewise, one can extend the above approach to capture the current and multiple previous outputs $\boldsymbol{v}_{l}, \ldots, \boldsymbol{v}_{l-m_{e x}}$ by introducing a generator matrix with multiple extended memories, i.e.,

$$
\boldsymbol{G}_{s}^{e x}=\left[\begin{array}{llll}
\boldsymbol{G}_{s} & D \boldsymbol{G}_{s} & \cdots & D^{m_{e x}} \boldsymbol{G}_{s}
\end{array}\right],
$$

where $m_{e x}$ corresponds to the size of external memory bits. An example encoder with $\nu=2$ and $m_{e x}=3$ is sketched in Fig. 2. Since $\boldsymbol{G}_{s}$ itself has $\nu$ bit memories, the overall bit memory size of $\boldsymbol{G}_{s}^{e x}$ is given by $\nu+m_{e x}$ and the number of states $\Gamma$ of the resulting trellis diagram is $2^{\nu+m_{e x}}$.

The VA is carried out on this extended trellis with the branch metric for the PAR reduction (which we will define in the next subsection). After the completion of the VA, the trace-back operation outputs codeword $\boldsymbol{v}$ along the determined path, and then the transmitted bits $\boldsymbol{z}$ are formed based on (4). Note that, although the $l$ th trellis section is associated with $\boldsymbol{v}_{l}$ and its previous counterparts $\boldsymbol{v}_{l-1}, \ldots, \boldsymbol{v}_{l-m_{e x}}$ for capturing the partial signal, only the output $\boldsymbol{v}_{l}$ is required for the $l$ th trellis section.

In order to describe the replica of the exact signals after pulse-shaping filter, the number of required external memories $m_{e x}$ should satisfy

$$
m_{e x}=\left\lceil K_{s} / d\right\rceil-1
$$

Therefore, as the length of effective impulse response $K_{s}$ increases, it causes a substantial increase of computational complexity. However, (18) also indicates that multidimensional shaping $(d>1)$ is effective to suppress the growth of complexity. For example, if $d=2, m_{e x}$ can be reduced by more than half compared with that of $d=1$. 


\section{B. Branch Metric Calculation}

Let $\Gamma^{\prime}$ and $\Gamma$ denote a pair of arbitrary states of the trellis diagram associated with the extended generator matrix $G_{s}^{e x}$ and suppose that the state transition $\Gamma^{\prime} \rightarrow \Gamma$ is valid for this code. Let

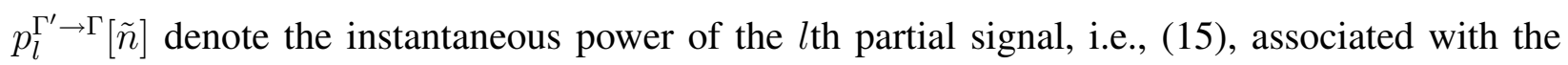
trellis state transition $\Gamma^{\prime} \rightarrow \Gamma$. Then the corresponding branch metric $B_{l}^{\Gamma^{\prime} \rightarrow \Gamma}$ can be defined by the following form

$$
B_{l}^{\Gamma^{\prime} \rightarrow \Gamma} \triangleq \sum_{k=0}^{d-1} \sum_{\tilde{n}=0}^{N_{s}-1} \mu\left(p_{d l+k}^{\Gamma^{\prime} \rightarrow \Gamma}[\tilde{n}]\right),
$$

where $\mu(\cdot)$ denotes a metric function with respect to each sample of the associated instantaneous power $p_{l}^{\Gamma^{\prime} \rightarrow \Gamma}[\tilde{n}]$. We consider the following two metric functions referred to as a limiter method and a moment method.

1) Limiter Method: Baseband signals are often clipped by soft envelope limiters prior to power amplification so as to avoid unacceptable nonlinear amplification. In such systems, the nonlinear distortion is characterized by the amount of the truncated part of the original signals. The limiter method thus tries to select a signal waveform candidate with minimum amount of energy that exceeds a certain threshold $p_{\max }$. Specifically, the metric function is given by

$$
\mu_{l m}(p)= \begin{cases}p-p_{\max }, & p>p_{\max } \\ 0, & p \leq p_{\max }\end{cases}
$$

2) Moment Method: It may be also effective to minimize the variance or other statistical moments of the output signal around its mean or certain reference level. The moment method aims at selecting the signal waveform with minimum value in terms of the $\beta$ th order central moment, i.e.,

$$
\mu_{m m}(p)=\left(p-p_{\text {ref }}\right)^{\beta}
$$

where $p_{\text {ref }}$ is a reference instantaneous power level. For PSK signals, it may be reasonable to set $p_{\text {ref }}$ around its mean, i.e., $p_{\text {ref }}=1$. As one can see, the order of statistical moment reflects various aspects of distributions, e.g., peakedness or smoothness. Thus, the choice of $\beta$ is capable of controlling the shape of the probability density function (pdf) of the resulting signal outputs and its associated PAR property. 


\section{Performance-Complexity Trade-off}

Our TS involves considerable computational effort, which stems mainly from the use of external memories and calculation of partial signals. In order to mitigate this requirement, the following approaches will be considered in this paper.

1) External Memory Reduction: From (18), the external memory $m_{e x}$ should be proportional to $K_{s}$ for accurate realization of the partial signals, and the parameter $K_{s}$ increases as a roll-off factor $\alpha$ becomes small. Apparently, one may curtail $m_{e x}$ to a smaller value than $\left\lceil K_{s} / d\right\rceil-1$, at the cost of reduced accuracy of partial signal representation. This may cause a loss in terms of PAR reduction capability.

2) Oversampling Factor Reduction: Unlike Viterbi decoder for channel coding, it is possible to compute summations of metric function, i.e., (19) for given bits $\left\{\boldsymbol{b}_{l}\right\}$, and tabulate them beforehand in our TS scenario. In this case, branch metrics can be obtained without any complex number operation. However, the necessary table size to store the computed metrics increases exponentially and it even becomes prohibitively large as $m_{e x}$ increases. One possible and appropriate alternative in such a situation is to store the oversampled versions of filtered symbol replica $\left\{S_{l} g[n]\right\}$ for all $S_{l} \in \mathcal{S}$ and calculate the partial signal at every branch by summing up appropriate portions of replicas. In this case, for a given oversampling factor $N_{s}$, every branch calculation involves $N_{s} d\left(1+m_{e x}\right)$ complex additions, $N_{s} d$ complex multiplications, and $N_{s} d$ times use of $\mu(\cdot)$ function, which dominate the overall complexity. Thus, decreasing $N_{s}$ may considerably alleviate computational effort with reduced accuracy of partial signal representation.

\section{Simulation Results}

We have performed simulations to evaluate the proposed trellis shaped PSK systems. The complementary cumulative distribution function (CCDF) of the instantaneous power normalized by the average power is obtained for evaluating signal dynamic range. Throughout the simulations, the PSK constellations are formed by Gray labeling, and a square-root raised-cosine filter for pulse shaping with a sufficient size of oversampling factor is used for evaluating continuous output signals. Note that the invariant property of the average power is guaranteed by Theorem 1. The duration of the impulse response $K_{s}$ varies depending on the roll-off factor $\alpha$, and we have chosen $K_{s}=12$ for $\alpha<0.4$, and $K_{s}=10$ for $\alpha \geq 0.4$. As an example, the shaping dimensionality $d$ is chosen to be either 1 or 2 . 
In the initial simulations, we use $N_{s}=8$ and $m_{e x}=K_{s} / d-1$ for the partial signal generation required for the trellis branch metric calculation. This parameter setting is sufficient to guarantee an accurate calculation of partial signals and therefore expected to serve as an ideal achievable performance.

\section{A. Information Rate and Choice of Shaping Encoder}

First, we determine the shaping encoder $\boldsymbol{G}_{s}$. Referring to (7), in the case of 8-PSK and $d=1$ (single dimensional shaping), one can choose either $n_{s}=2$ or 3 . For each $n_{s}$, we have performed an exhaustive computer search with memory sizes up to $\nu=3$ and found that the following parameters result in the best performance in terms of PAR reduction capability.

$$
\begin{aligned}
& \boldsymbol{G}_{s}=\left[\begin{array}{ll}
1+D^{3} & 1+D^{2}+D^{3}
\end{array}\right], \quad \text { for } n_{s}=2, \\
& \boldsymbol{G}_{s}=\left[\begin{array}{lll}
1+D^{3} & 1+D+D^{2}+D^{3} & D
\end{array}\right], \quad \text { for } n_{s}=3 .
\end{aligned}
$$

The corresponding syndrome formers and its left inverse for the above $n_{s}=3$ case are given by

$$
\begin{aligned}
\boldsymbol{H}_{s}^{T} & =\left[\begin{array}{cc}
D & 0 \\
0 & D \\
1+D^{3} & 1+D+D^{2}+D^{3}
\end{array}\right] \\
\left(\boldsymbol{H}_{s}^{-1}\right)^{T} & =\left[\begin{array}{lll}
1 & 0 & 0 \\
0 & 1 & 0
\end{array}\right]
\end{aligned}
$$

Note that the combination of the above matrices results in

$$
\left(\boldsymbol{H}_{s}^{-1}\right)^{T} \boldsymbol{H}_{s}^{T}=D \boldsymbol{I},
$$

which implies that one symbol delay occurs at the receiver.

Simulation results (not shown here for space limitation) suggest that the CCDFs of the instantaneous power with $n_{s}=2$ and 3 show similar behavior, but in some cases the encoder with $n_{s}=3$ shows slightly better performance. In the case of $d=2$ (two dimensional shaping), the following encoder with $n_{s}=6$

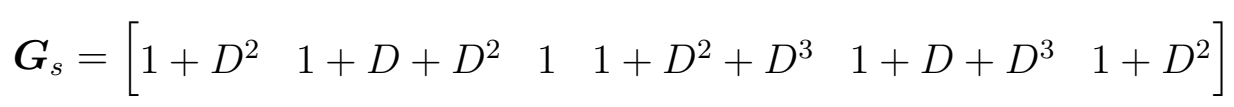

has shown a good performance. In the following simulations, $n_{s}=3$ and 6 are chosen for $d=1$ and 2, respectively. It can be seen from (5) that in these cases, all the information bits $b$ are 
fed to the inverse syndrome. Hence, the non-shaping bits $\boldsymbol{u}$ are absent. This means that the following results do not depend on a particular choice of constellation labeling.

\section{B. Limiter Method and Moment Method}

The CCDFs for the limiter and moment methods are plotted in Figs. 3 and 4, respectively, along with those of $\pi / 4$-shift QPSK and the conventional TS with a branch metric equal to the phase difference (with the information rate being 2 [bit/symbol]) as proposed in [4]. For demonstration purpose, the results are shown with several different parameter settings. Fig. 5 shows the relationship between the roll-off factor $\alpha$ and the resulting instantaneous power level at $\mathrm{CCDF}=10^{-5}$. We observe the following remarks from these results.

- The shaped dynamic range depends on the metric parameters $\left(p_{\max }\right.$ or $\beta$ ). A similar tendency in terms of CCDF characteristic is observed for the two methods. By definition, the limiter method of (20) minimizes the probability that the instantaneous power exceeds the threshold $p_{\text {max }}$, which suggests that the CCDF value at the reference threshold power (abscissa) equal to $p_{\max }$ is minimized. The choice of parameter setting, however, depends on the system requirement such as within which range of CCDF one should minimize the threshold power. In this case, the appropriate value of $p_{\max }$ should be determined by simulations. On the other hand, in the moment method, there is no explicit relationship between the parameter $\beta$ and the resulting CCDF. We have confirmed by simulation that setting $\beta=8$ can obtain the minimum threshold power level evaluated at $\mathrm{CCDF}=10^{-5}$.

- Fig. 5 suggests that the proposed TS can achieve very low PAR throughout all simulated values of $\alpha$, and the limiter method is always superior to the moment method approximately by 0.2 to $0.5 \mathrm{~dB}$.

- The use of multidimensional shaping $(d=2)$ offers a trade off in terms of enhanced information rate by 0.5 [bit/symbol] and increased PAR about $1 \mathrm{~dB}$ compared to the single dimensional shaping $(d=1)$. Substantial PAR reduction compared to unshaped system is still observed.

Fig. 6 shows example signal trajectories of baseband waveforms with and without shaping for 8-PSK. For the shaped waveform, we set $d=1$ and employed the limiter method with $p_{\max }=$ 1.33. As observed, the proposed shaping exhibits near constant envelope. Since the proposed trellis shaping actually generates the pulse-shaped signals for branch metric calculation, it is also 


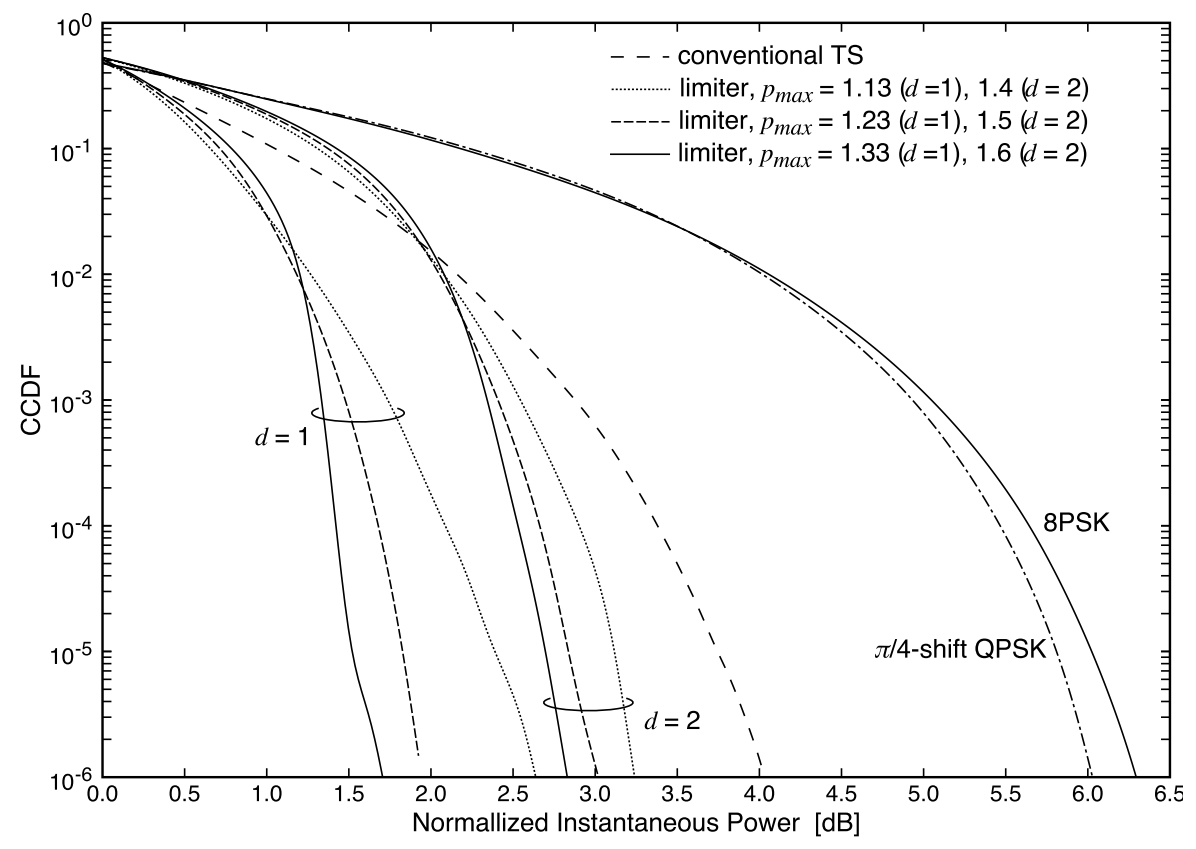

Fig. 3. $\mathrm{CCDF}$ of the instantaneous power based on the limiter method with several different values of $p_{\max }\left(\alpha=0.1, N_{s}=8\right.$, and $m_{e x}=11$ and 6 for $d=1$ and 2 , respectively). Note that these parameters $m_{e x}$ and $N_{s}$ are chosen large enough to generate accurate partial signals.

effective for constellations with orders higher than 8-PSK. For example, we have confirmed by simulation that 32-PSK baseband waveforms have almost the same signal trajectories as that of 8-PSK shown in Fig. 6. On the other hand, the conventional techniques based on the control of phase difference among successive symbols [4] have limited capability in reducing envelope fluctuation as the modulation order increases. Note that applying the proposed TS to higher order constellations does not significantly increase the overall system complexity, as (18) indicates no dependence of the order of modulation $M$ on the trellis size.

\section{Effects of External Memory and Oversampling Factor}

Fig. 7 shows an achievable instantaneous power level at $\mathrm{CCDF}=10^{-5}$ when the number of the external memory $m_{e x}$ is reduced. From this figure, we can observe that compared to the moment method, the limiter method is more sensitive to the length of memory, or, in other words, inaccuracy of the partial waveforms representation. A similar behavior is also observed when the oversampling factor $N_{s}$ is reduced. Fig. 8 shows the threshold instantaneous power 


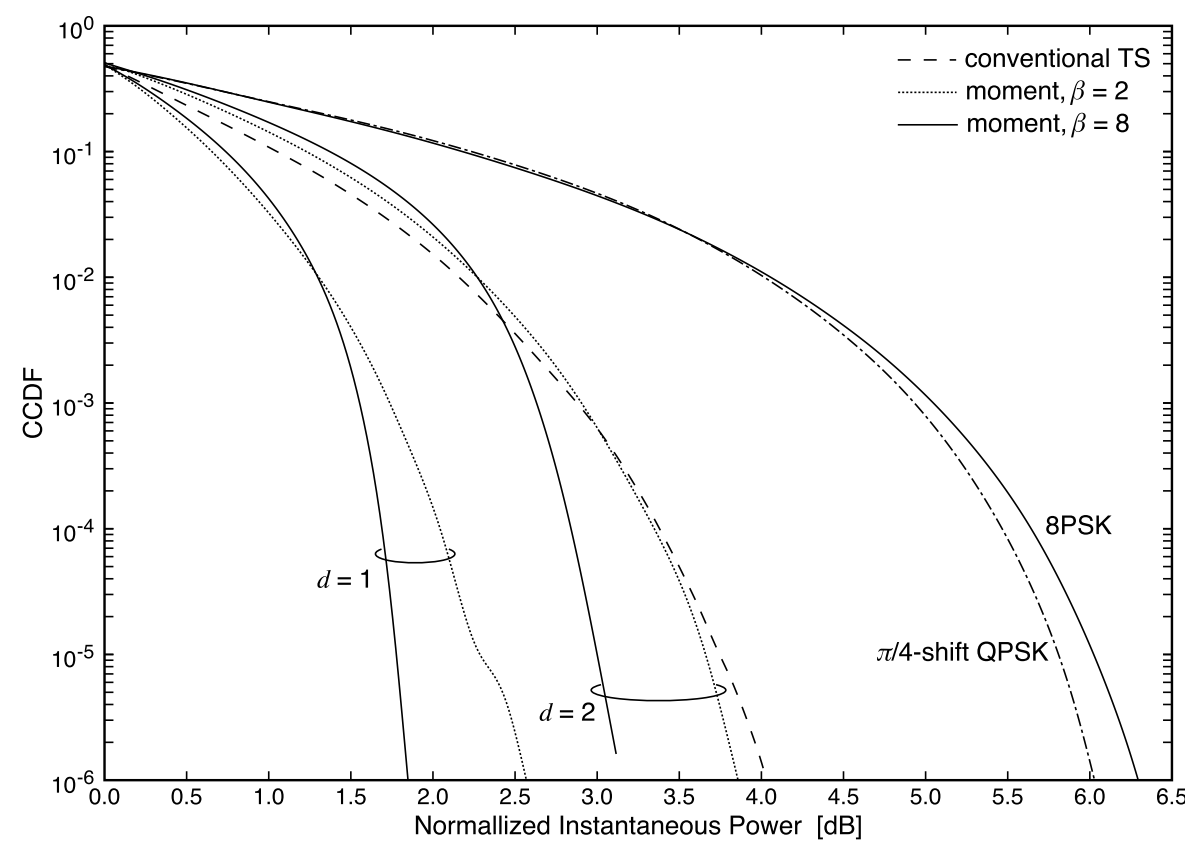

Fig. 4. CCDF of the instantaneous power based on the moment method with $\beta$ being a parameter $(\alpha=0.1)$. Parameter settings of $N_{s}$ and $m_{e x}$ are identical to those of Fig. 3.

at $\mathrm{CCDF}=10^{-5}$ for a given $N_{s}$. It is interesting to observe that the moment method has no performance degradation even with $N_{s}=2$, whereas the limiter method demonstrates a noticeable increase of instantaneous power level.

Another remarkable aspect observed from Fig. 7 is that with $\alpha=0.1$, the multidimensional shaping performs similar to or even better than the single dimensional shaping when the size of external memory is restricted to a small value. This is due to the fact that for a fixed value of $m_{e x}$, the multidimensional shaping can work with more accurate partial signals than the single dimensional shaping as inferred from (18). For example, in the case of $\alpha=0.1$, the proposed TS even with $d=2$ and $m_{e x}=4$ is shown to reduce the instantaneous power level by more than $3 \mathrm{~dB}$ compared to $\pi / 4$-QPSK. Furthermore, in this case, the information rate is by 0.5 [bit/symbol] higher than that of $\pi / 4$-QPSK, and the shaping trellis has only $2^{\nu+m_{e x}}=2^{3+4}=128$ states, which is less than those typically used for error correction in the recent mobile communications standards.

Finally, we note that the single dimensional shaping with $m_{e x}$ close to $K_{s}$ may be still a valid 


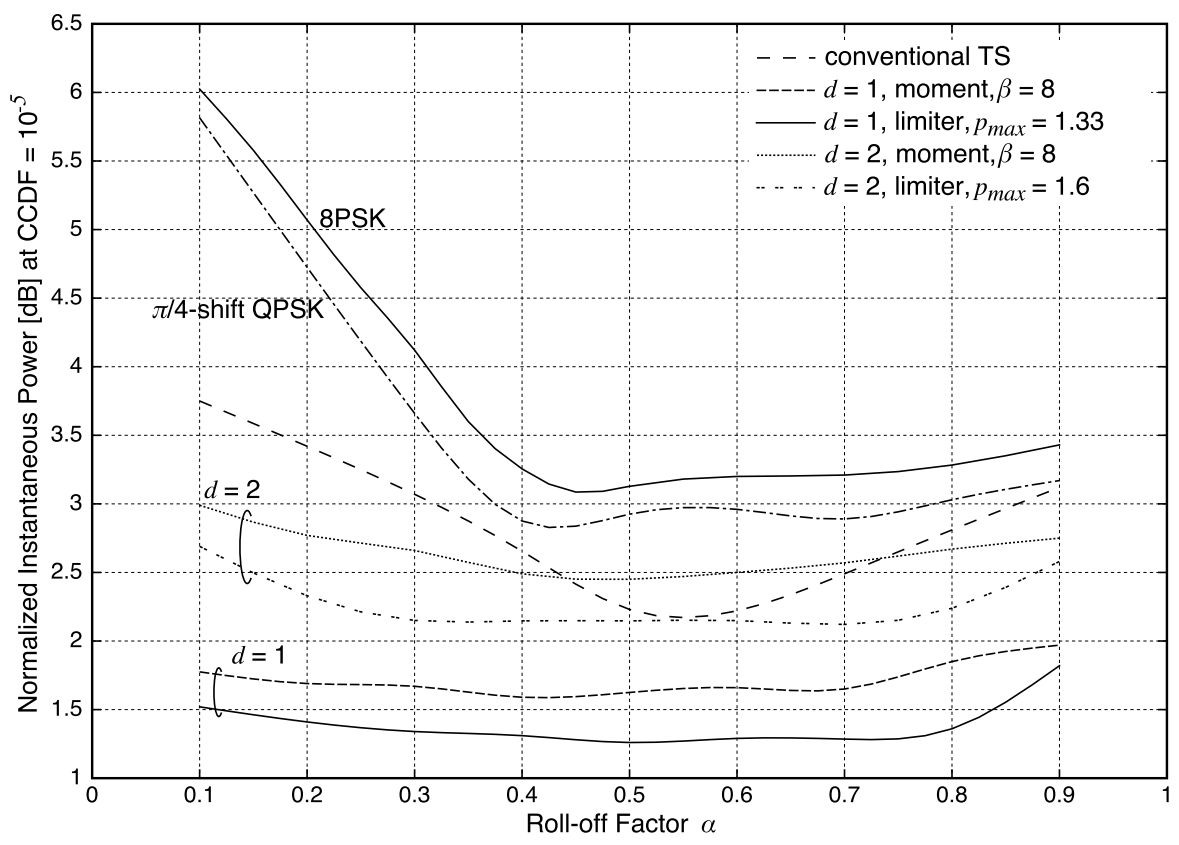

Fig. 5. Normalized instantaneous power at $\mathrm{CCDF}=10^{-5}$ versus roll-off $\alpha$.

option for deep space and satellite communications where the PA efficiency is one of the most important factors, considering that the Viterbi decoder with the number of states as large as $2^{14}$ is in fact developed for such applications [10].

\section{Efficiency of Trellis Shaping}

The reduction of the information rate is one of the major drawbacks of the TS approach. When $M$-ary PSK is used, the total information rate of our TS is reduced by $1 / d$ bits per symbol just for constraining the symbol sequence. In order to justify this rate loss, the achievable upper bound of the information rate, or entropy, given the symbol constraint achieved by the TS, is of particular interest. Therefore, in what follows, we investigate the gap between $R_{s}$ and the entropy (per one transmitted symbol) of the observed symbols after shaping, denoted by $H(\boldsymbol{S})$. For this purpose, we define the shaping efficiency as

$$
\eta_{s} \triangleq \frac{R_{s}}{H(\boldsymbol{S})} \times 100 \quad[\%]
$$

In general, $H(\boldsymbol{S})$ can be calculated from $N$-dimensional joint probability where $N$ is infinitely 


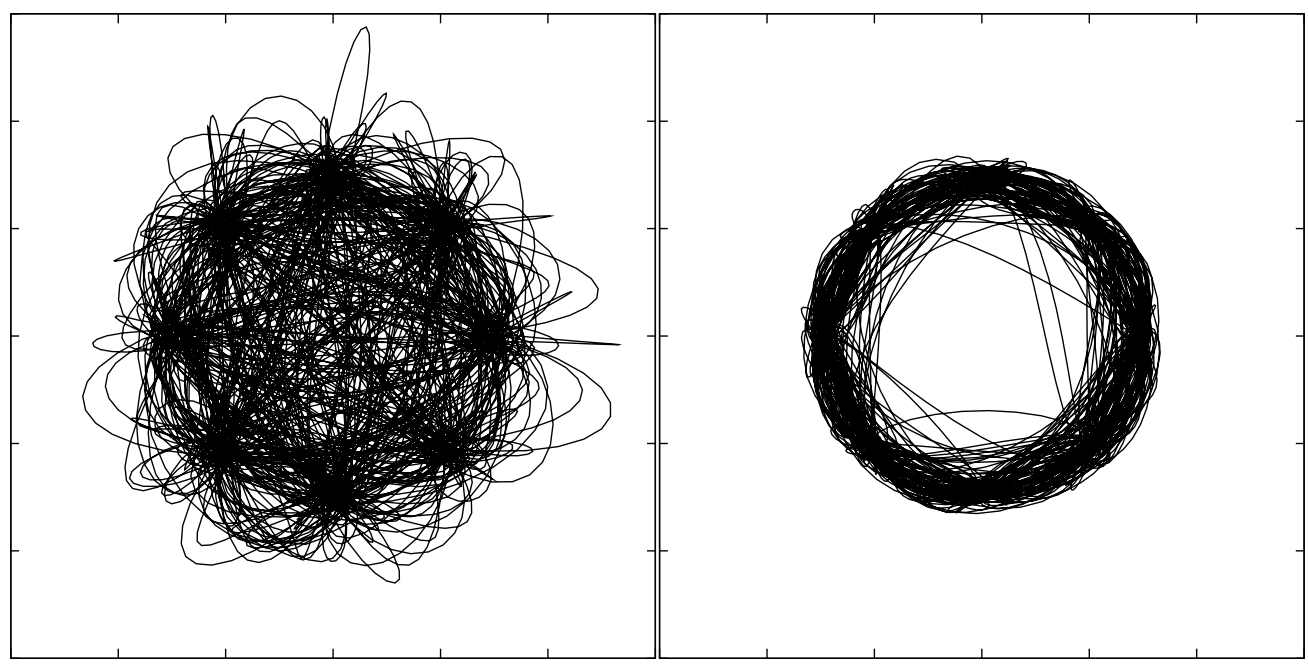

Fig. 6. Signal trajectories of baseband waveform $(\alpha=0.1)$. Left: unshaped 8-PSK. Right: shaped 8-PSK with limiter method $p_{\max }=1.33$.

large, i.e.,

$$
H(\boldsymbol{S})=-\lim _{N \rightarrow \infty} \frac{1}{N} E\left[\log _{2} \operatorname{Pr}\left(S_{1}, S_{2}, \cdots, S_{N}\right)\right]
$$

In order to make the above entropy numerically tractable, we use the following decomposition:

$$
\operatorname{Pr}\left(S_{1}, S_{2}, \cdots, S_{N}\right)=\operatorname{Pr}\left(S_{N} \mid S_{N-1}, \cdots, S_{1}\right) \operatorname{Pr}\left(S_{N-1} \mid S_{N-2}, \cdots, S_{1}\right) \cdots,
$$

and approximate the conditional probabilities by assuming that the shaped sequence forms a Markov chain with a finite order. Specifically, we assume

$$
\operatorname{Pr}\left(S_{l} \mid S_{l-1}, \cdots, S_{1}\right)=\operatorname{Pr}\left(S_{l} \mid S_{l-1}, \cdots, S_{l-m_{m}}\right)
$$

where $m_{m} \geq 1$ is the order of the Markov chain. Then we investigate the convergence behavior of $H(\boldsymbol{S})$ with increasing $m_{m}$.

As an example, we have numerically evaluated (29) for the trellis shaped outputs with $\alpha=0.4$ and $K_{s}=10$ (the conditional probabilities are also calculated by computer simulation) and obtained the following results: with 8-PSK, the information rate of TS system with $d=1$ is 2 [bit/symbol], whereas the entropies for $m_{m}=1,2,3$, and 4 are calculated as 2.26, 2.13, 2.09, and 2.07, respectively. The shaping efficiency evaluated with $m_{m}=4$ is $96[\%]$. Note that the efficiency is expected to be slightly higher as we further increase $m_{m}$. 


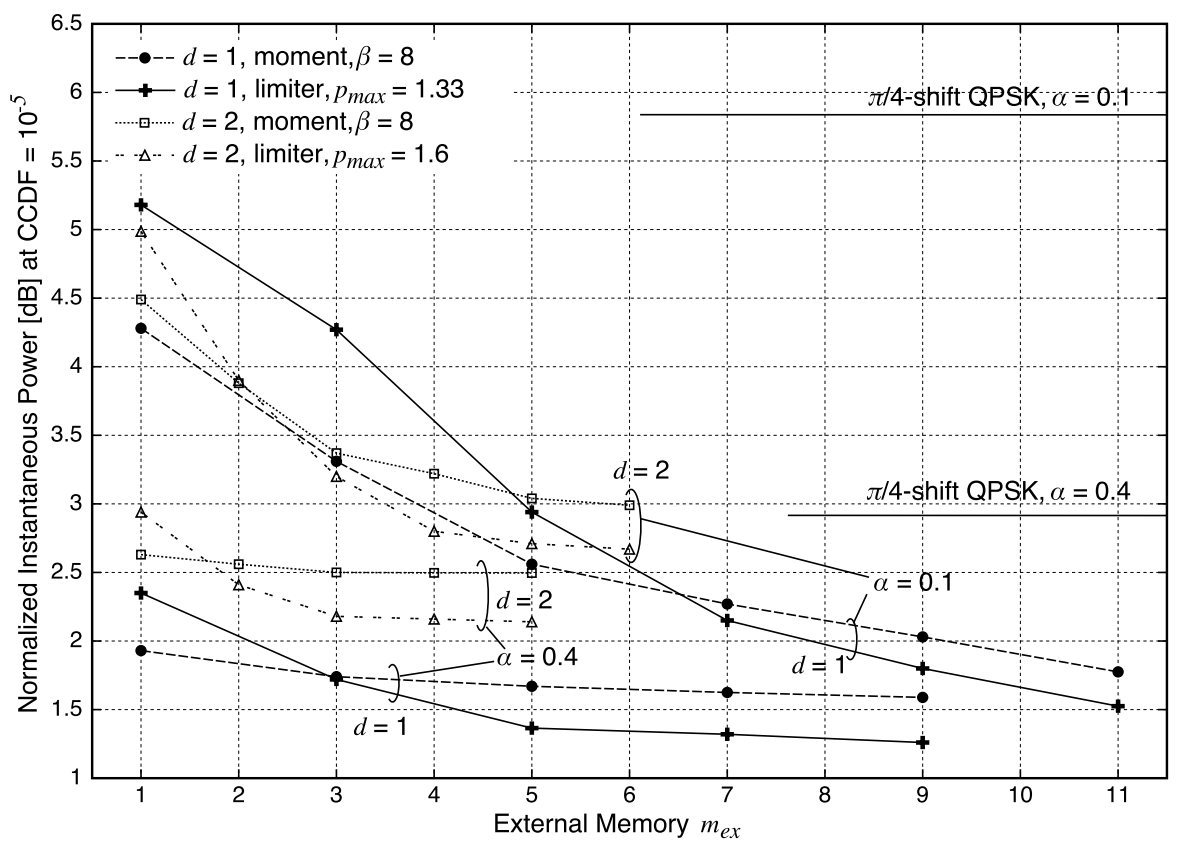

Fig. 7. Normalized instantaneous power at CCDF $=10^{-5}$ versus the size of external memory. Achievable values of $\pi / 4$-shift QPSK constellation are also plotted.

To conclude, the gap between the entropy and the actual information rate may be negligibly small. This indicates that the proposed TS is effective in terms of achievable information rate for a given symbol transition constraint.

\section{E. Robustness against Decision Timing Jitter}

One of major drawbacks of the use of pulse-shaping filter with low roll-off factor is its sensitivity to the ISI caused by the sampling timing jitter at the receiver side. However, since the proposed TS reduces the fluctuation of signal envelope around sampling instants, it exhibits strong robustness against the timing jitter. As an example, Fig. 9 shows constellations of 8-PSK sampled with a fixed timing jitter $\Delta T=T_{s} / 8$, where $T_{s}$ is the Nyquist interval, with and without the proposed shaping. As observed, without shaping, the detected symbols at the receiver scatters both in the amplitude and phase, whereas only the phase disturbance dominates for the case with the proposed shaping. Fig. 10 shows the corresponding symbol error rate (SER) versus $E_{s} / N_{0}$ where $E_{s}$ denotes the received symbol energy and $N_{0}$ is a one-sided power spectral density of the additive white Gaussian noise. We observe a severe error floor in the case without shaping for 


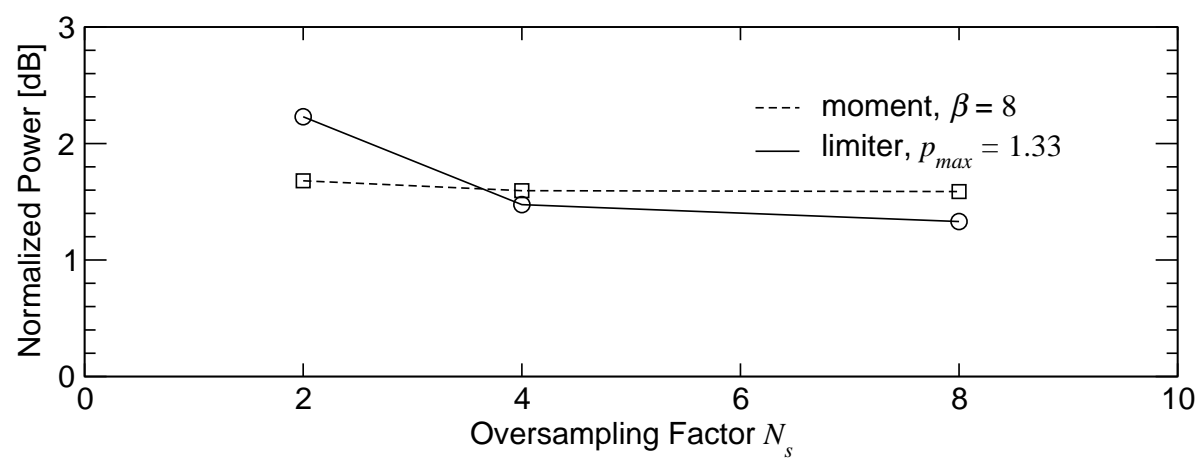

Fig. 8. Normalized instantaneous power at $\mathrm{CCDF}=10^{-5}$ versus oversampling factor $(d=1)$.

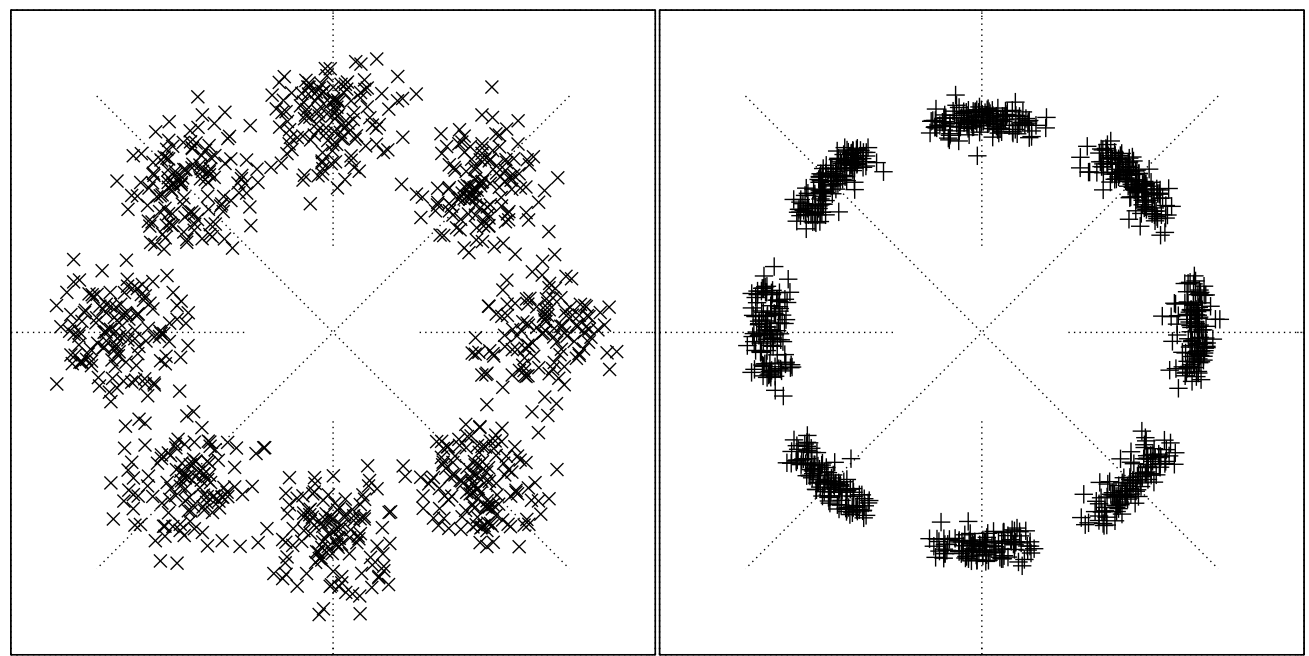

Fig. 9. Symbol constellation at the receiver with timing error $\Delta T=T_{s} / 8$. Left: unshaped. Right: shaped.

the system with $\alpha=0.1$, whereas no error floor is found in the case with shaping. Consequently, with the proposed TS the requirement for the receiver timing synchronization accuracy may be significantly mitigated.

\section{CONCLUSION}

In this paper, we have proposed a powerful trellis shaping approach for PAR reduction of single-carrier PSK signals. Computer simulations have demonstrated that the proposed TS can achieve almost constant envelope.

Our method consists of partial signal generation and the two metric functions. Both the metric functions enjoy adjustability of CCDF characteristics with their associated parameters (threshold 


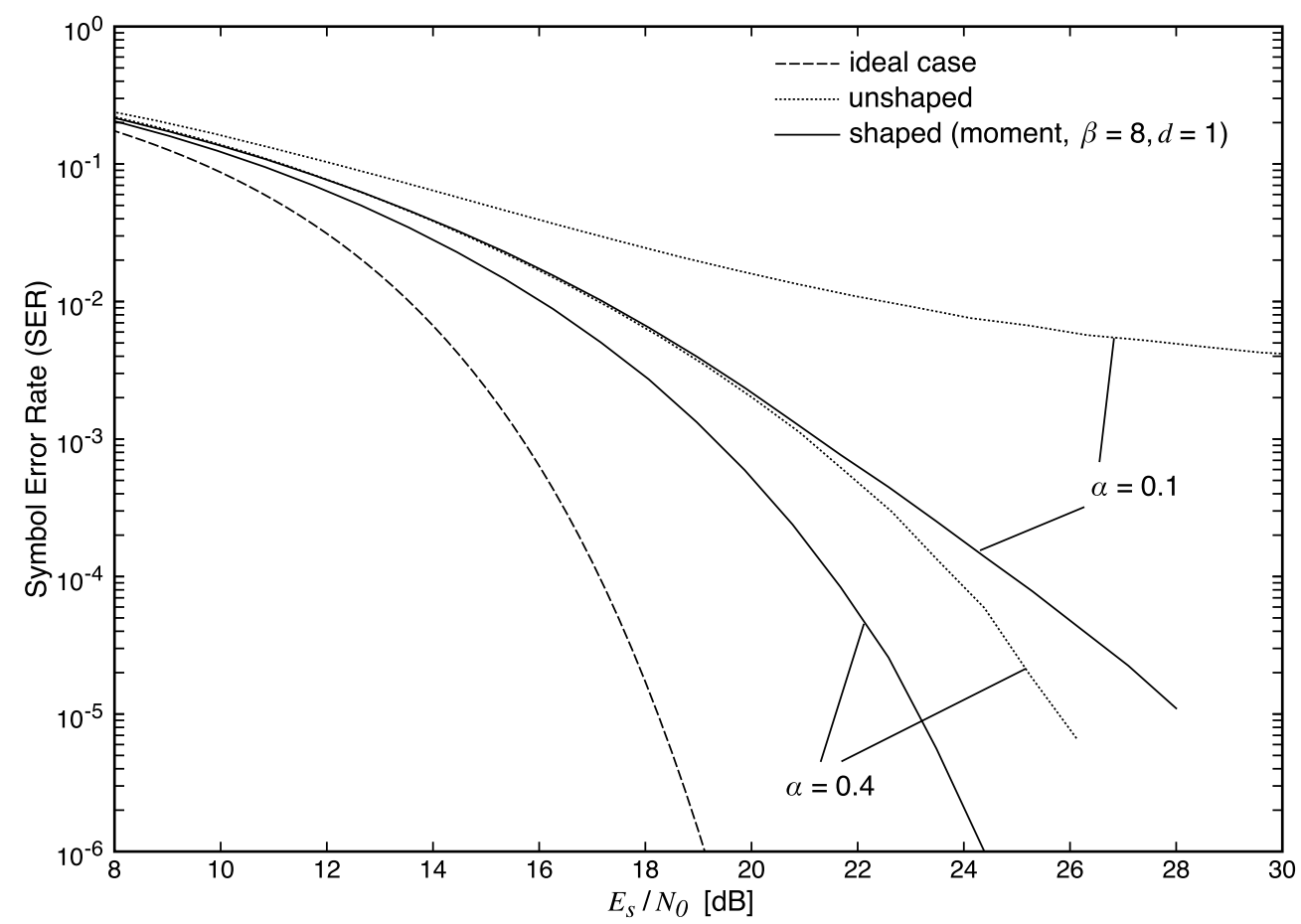

Fig. 10. Symbol error rate versus $E_{s} / N_{0}$ with timing error $\Delta T=T_{s} / 8$.

level $p_{\max }$ or moment order $\beta$ ). Simulation results have shown that the limiter method has better PAR control capability, but is also sensitive to the accuracy of the partial signals. Therefore, the complexity requirement of the limiter method may be higher than that of the moment method. Finally, we note that the proposed method is also applicable to PAR reduction of high-order QAM, with some suitable modification of metric functions.

\section{APPENDIX}

\section{A. Proof of Theorem 1}

For a given stationary sequence $\left\{S_{l} \mid-\infty<l<\infty\right\}$ with Nyquist interval $T_{s}$, the pulse-shaped signal is expressed as

$$
s(t)=\sum_{l=-\infty}^{\infty} S_{l} g\left(t-l T_{s}\right)
$$


where $g(t)$ denotes the impulse response of the filter. From the first condition, without loss of generality we assume that the energy of the impulse response is normalized such that

$$
\int_{-\infty}^{\infty}|g(t)|^{2} d t=1
$$

We can also assume that $g(t)$ has a finite effective length, i.e.,

$$
\lim _{t \rightarrow \infty} g(t)=\lim _{t \rightarrow-\infty} g(t)=0 .
$$

The autocorrelation function of $s(t)$ is expressed as

$$
\begin{aligned}
\phi(t, \tau) & \triangleq E\left[s^{*}(t+\tau) s(t)\right] \\
& =\sum_{k=-\infty}^{\infty} \sum_{n=-\infty}^{\infty} \phi_{S}[k-n] g^{*}\left(t+\tau-k T_{s}\right) g\left(t-n T_{s}\right),
\end{aligned}
$$

where $\phi_{S}[l]=E\left[S_{n+l}^{*} S_{n}\right]$ denotes the autocorrelation function of $\left\{S_{l}\right\}$. It can be easily confirmed that (35) satisfies cyclostationarity:

$$
\phi(t, \tau)=\phi\left(t+T_{s}, \tau\right)
$$

It is thus sufficient to consider the autocorrelation function within the period $T_{s}$. Let $\bar{\phi}(\tau)$ denote the time average autocorrelation function [9], which can be calculated as

$$
\begin{aligned}
\bar{\phi}(\tau) & =\frac{1}{T_{s}} \int_{0}^{T_{s}} \phi(t, \tau) d t \\
& =\frac{1}{T_{s}} \sum_{k=-\infty}^{\infty} \sum_{n=-\infty}^{\infty} \phi_{S}[k-n] \int_{-k T_{s}}^{(1-k) T_{s}} g^{*}(t+\tau) g\left(t+(k-n) T_{s}\right) d t .
\end{aligned}
$$

Let $X[k]$ denote the summand of the outer summation, i.e.,

$$
X[k] \triangleq \sum_{n=-\infty}^{\infty} \phi_{S}[k-n] \int_{-k T_{s}}^{(1-k) T_{s}} g^{*}(t+\tau) g\left(t+(k-n) T_{s}\right) d t .
$$

For $k=0$, we have

$$
X[0]=\sum_{n=-\infty}^{\infty} \phi_{S}[-n] \int_{0}^{T_{s}} g^{*}(t+\tau) g\left(t-n T_{s}\right) d t
$$

and for $k=1$, we have

$$
\begin{aligned}
X[1] & =\sum_{n=-\infty}^{\infty} \phi_{S}[1-n] \int_{-T_{s}}^{0} g^{*}(t+\tau) g(t+(1-n) T s) d t \\
& =\sum_{n=-\infty}^{\infty} \phi_{S}[-n] \int_{-T_{s}}^{0} g^{*}(t+\tau) g(t-n T s) d t,
\end{aligned}
$$


where we have replaced $n-1$ with $n$. Likewise, for any finite integer $k$, we have

$$
X[k]=\sum_{n=-\infty}^{\infty} \phi_{S}[-n] \int_{-k T_{s}}^{(1-k) T_{s}} g^{*}(t+\tau) g\left(t-n T_{s}\right) d t .
$$

Due to the assumption of (34), it can be shown that $\lim _{k \rightarrow \infty} X[k]=0$. Consequently,

$$
\begin{aligned}
\bar{\phi}(\tau) & =\frac{1}{T_{s}} \sum_{k=-\infty}^{\infty} X[k] \\
& =\frac{1}{T_{s}} \sum_{n=-\infty}^{\infty} \phi_{S}[-n] \sum_{k=-\infty}^{\infty} \int_{-k T_{s}}^{(1-k) T_{s}} g^{*}(t+\tau) g\left(t-n T_{s}\right) d t \\
& =\frac{1}{T_{s}} \sum_{n=-\infty}^{\infty} \phi_{S}^{*}[n] \int_{-\infty}^{\infty} g^{*}(t+\tau) g\left(t-n T_{s}\right) d t .
\end{aligned}
$$

Once we obtain the (time average) autocorrelation function $\bar{\phi}(\tau)$, the average power $P_{a v}$ is given by setting $\tau=0$, i.e.,

$$
P_{a v}=\bar{\phi}(0)=\frac{1}{T_{s}} \sum_{n=-\infty}^{\infty} \phi_{S}^{*}[n] \int_{-\infty}^{\infty} g^{*}(t) g\left(t-n T_{s}\right) d t .
$$

By the even function property of $g(t)$, i.e.,

$$
g(-t)=g(t)
$$

and the Nyquist condition for the zero ISI, i.e.,

$$
\int_{-\infty}^{\infty} g^{*}(t) g\left(n T_{s}-t\right) d t=\delta[n]
$$

where $\delta[n]$ denotes the Kronecker delta function, we obtain

$$
P_{a v}=\frac{1}{T_{s}} \sum_{n=-\infty}^{\infty} \delta[n] \phi_{S}[n]=\frac{1}{T_{s}} \phi_{S}[0] .
$$

Hence, regardless of correlation properties of $\left\{S_{l}\right\}, P_{a v}$ depends only on the average energy of discrete symbol, $\phi_{S}[0]=E\left[\left|S_{l}\right|^{2}\right]$, which is always constant for PSK constellations.

\section{REFERENCES}

[1] S. L. Miller and R. J. O’Dea, "Peak power and bandwidth efficient linear modulation," IEEE Trans. Commun., vol. 46, pp. 1639-1648, Dec. 1998.

[2] M. Chen and O. M. Collins, “Trellis pruning for peak-to-average power ratio reduction,” in Proc. IEEE ISIT’2005, pp. 12611265, Sept. 2005.

[3] G. D. Forney, Jr., “Trellis shaping,” IEEE Trans. Inform. Theory, vol. 38, pp. 281-300, Mar. 1992. 
[4] I. S. Morrison, "Trellis shaping applied to reducing the envelope fluctuations of MQAM and band-limited MPSK," in Proc. Int. Conf. Digital Satellite Commun. (ICDSC'92), pp. 143-149, May 1992.

[5] M. Litzenburger and W. Rupprecht, "Combined trellis shaping and coding to control the envelope of a bandlimited PSKsignal,” in Proc. IEEE ICC’94, pp. 630-634, June 1994.

[6] W. Henkel and B. Wagner, "Another application for trellis shaping: PAR reduction for DMT (OFDM)," IEEE Trans. Commun., vol. 48, pp. 1471-1476, Sept. 2000.

[7] H. Ochiai, "A novel trellis-shaping design with both peak and average power reduction for OFDM systems," IEEE Trans. Commun., vol. 52, pp. 1916-1926, Nov. 2004.

[8] R. F. H. Fischer, Precoding and Signal Shaping for Digital Transmission. John Wiley \& Sons Inc, 2002.

[9] J. G. Proakis, Digital Communications. McGrawHill, 4th ed., 2001.

[10] O. M. Collins, "The subtleties and intricacies of buliding a constraint length 15 convolutional decoder," IEEE Trans. Commun., vol. 40, pp. 1810-1819, Dec. 1992.

\begin{tabular}{|c|}
\hline \\
PLACE \\
PHOTO \\
HERE \\
\hline
\end{tabular}

Makoto Tanahashi (S'07) was born in Gifu, Japan, on December 20, 1983. He received the B.E. and M.E. degrees in electrical engineering from the Yokohama National University, Japan, in 2006 and 2008, respectively. He is currently working toward the Ph.D. degree in electrical engineering at the Yokohama National University. 